\title{
Effects of Composite Supplement Containing Astaxanthin and Sesamin on Cognitive Functions in People with Mild Cognitive Impairment: A Randomized, Double-Blind, Placebo-Controlled Trial
}

\author{
Naoki Ito ${ }^{\mathrm{a}, *}$, Hitomi Saito ${ }^{\mathrm{a}}$, Shinobu Seki ${ }^{\mathrm{a}}$, Fumitaka Ueda ${ }^{\mathrm{a}}$ and Takashi Asada ${ }^{\mathrm{b}}$ \\ ${ }^{a}$ Pharmaceutical and Healthcare Research Laboratories, Research and Development Management \\ Headquarters, FUJIFILM Corporation, Ashigarakami-gun, Kanagawa, Japan \\ ${ }^{\mathrm{b}}$ Memory Clinic Ochanomizu, Bunkyo-ku, Tokyo, Japan
}

Accepted 11 January 2018

\begin{abstract}
.
Background: Dementia and its first or transitional stage, mild cognitive impairment (MCI), is a major concern for the aging Japanese society. Thus, the use of dietary supplements to improve or maintain cognitive function has become a topic of public interest.

Objective: In this study, we evaluated the effects of a composite supplement containing food-derived antioxidants, specifically astaxanthin and sesamin (AS), on cognitive function in people with MCI.

Method: Twenty-one healthy participants with MCI were recruited in our double-blind placebo-controlled pilot study. They were assigned to either an AS group, who received ingestible capsules containing AS, or a placebo group, who received identical placebo capsules. To assess cognitive functions, we performed the Japanese version of the Central Nervous System Vital Signs (CNSVS) test and the Alzheimer's Disease Assessment Scale-Cog test at baseline, after 6 weeks, and after 12 weeks of dietary supplementation.

Results: The CNSVS test revealed significant improvements in psychomotor speed and processing speed in the AS group compared with the placebo group, suggesting that the daily supplementation of AS improved cognitive functions related to the ability to comprehend, and perform complex tasks quickly and accurately.

Conclusion: Our results provide support for the use of AS as a dietary supplementation for improving cognitive functions.
\end{abstract}

Keywords: Astaxanthin, CNSVS, cognitive functions, mild cognitive impairment, sesame extract, sesamin

\section{INTRODUCTION}

According to the World Health Organization, approximately 47 million people worldwide suffer

\footnotetext{
${ }^{*}$ Correspondence to: Naoki Ito, Pharmaceutical and Healthcare Research Laboratories, Research and Development Management Headquarters, FUJIFILM Corporation, 577, Ushijima, Kaiseimachi, Ashigarakami-gun, Kanagawa 258-8577, Japan. E-mail: naoki.a.ito@fujifilm.com.
}

from dementia, with 9.9 million new cases every year [1]. Alzheimer's disease (AD) is the most common cause of dementia. AD is a neurodegenerative disease with many characteristic and pathological features, though the exact causes and mechanisms underlying $\mathrm{AD}$ progression are still controversial. Cognitive impairments that are insufficiently severe to warrant a diagnosis of dementia are referred to as mild cognitive impairment (MCI). MCI is described as the first 
stage or the transitional stage of dementia, because people with MCI develop AD at a rate of $10-15 \%$ every year [2]. Numerous new treatments are being investigated, and these are in various stages of clinical trials. However, to date, no effective cures for AD or MCI have been established.

Oxidative stress is thought to be involved in the pathogenesis and progression of age-related cognitive impairments [3]. Because anti-oxidative capacity decreases with age [4], the prevention and treatment of cognitive impairments by food-derived antioxidants are highlighted [5]. Astaxanthin is a red carotenoid found in salmon, shrimp, crab, and microalgae [6, 7]. Astaxanthin exerts a strong antioxidative effect by scavenging free radicals such as singlet oxygen [8]. Ingested astaxanthin is absorbed in the small intestine, and reaches the plasma and erythrocytes [9], epidermis and dermis [10], and brain by crossing the blood-brain barrier [11]. The strong anti-oxidative activity of astaxanthin has inspired researchers to evaluate the beneficial effects of astaxanthin as a supplement for wide variety of human health factors including skin conditions [12], metabolism [13, 14], exercise performance [15], and sleep efficiency (in combination with zinc) [16]. The beneficial effects of astaxanthin for brain function have been investigated in both mice and humans [17-19]. Astaxanthin has been found to show neuroprotective effects. Specifically, it reduces oxidative stress in the brain, and is thought to alleviate oxidative stress-related brain dysfunctions. Sesame is a major component in Asian cuisine [20]. Similar to astaxanthin, treatment with sesamin, which is a major lignan found in sesame extract, also produced neuroprotective effects, and has been found to alleviate the cerebral ischemia-induced damage [21, 22].

The beneficial effects of astaxanthin for people with dementia or MCI are still controversial though few studies have evaluated the effects of dietary supplementation with astaxanthin on cognitive function in humans. The effects of astaxanthin on the improvement of cognitive function have been previously evaluated by other group [17]. Although differences between supplementation with astaxanthin and that with placebo were not observed, statistically significant improvement of cognitive function from baseline by supplementation with astaxanthin was observed, which encouraged us to evaluate the combined supplementation with some ingredient which would enhance the effect of astaxanthin. Furthermore, to our knowledge, the effects of sesamin on cognitive function have not been investigated in humans. Several lines of evidence have shown that the absorption and tissue distribution of astaxanthin is different from that of sesamin, suggesting that the beneficial effects of the two compounds are not equivalent [23-25]. Astaxanthin was absorbed with peak plasma concentration at 5 hours [26], while sesamin and its metabolite was absorbed with relatively quick peak at one hour [24]. We hypothesized that the quickly absorbed sesamin reduced the systemic oxidative stress, which could protect astaxanthin from the immediate degradation and eventually brought out much stronger anti-oxidative capacity. In this study, we evaluated the combined effects of dietary supplementation with astaxanthin and sesamin (AS) on a wide range of cognitive functions in people with MCI. We used the Japanese version of the Central Nervous System Vital Signs (CNSVS, also known as 'Cognitrax' in Japan) computerized battery test [27] and the Alzheimer's Disease Assessment Scale-Cog (ADAS$\mathrm{Cog}$ ) as the primary outcomes [28]. As secondary outcomes, we examined blood elements related to oxidative stress and brain-derived neurotrophic factor (BDNF) levels, and also conducted a safety evaluation.

\section{MATERIALS AND METHODS}

\section{Study design, randomization, and blinding}

This study was a randomized, double-blind, placebo-controlled, parallel-group comparison trial conducted to evaluate the effects of dietary supplementation with AS on cognitive function in MCI participants. We allocated an equal number of participants to the placebo and active groups. The study was approved by the Ethical Committee of Nihonbashi Cardiology Clinic (Approved Number: NJI-016-0701). This study was registered in the UMIN Clinical Trials Registry (ID: UMIN000023391). The study protocol adhered to the Declaration of Helsinki and the Ethical Guidelines for Medical and Health Research Involving Human Subjects. Participants, practitioners, and clinicians were blinded. Clinicians performed the intervention and outcome measurements. Practitioners performed the analysis. As long as we examined, the minimum number of participants required to evaluate the intervention effects using the CNSVS test was 10 [29]. According to our previous independent trials conducted on 10 healthy people [30], we set the required sample size as 10 . 
We set the CNSVS and ADAS-cog tests as the primary outcomes. We also set the safety evaluation and the blood test for malondialdehyde (MDA), oxidized low-density lipoprotein (ox-LDL), serum total antioxidant status (STAS), paraoxonase 1 (PON-1), and BDNF as the secondary outcomes. Because eligible participants were serially incorporated into the study, participants were assigned to the AS or placebo group using an alternating quasi-randomization allocation method. Allocation was concealed until all participants finished the tests. The one who was different from the controller of this study enrolled and assigned the participants according to sex, age, and score on the Hierarchic Dementia Scale-Revised (HDS-R). The participants were enrolled by the responsible doctor.

\section{Participants}

This study included participants aged from 50 to 79 years old in the Tokyo metropolitan area who attended Memory Clinic Ochanomizu, and were diagnosed with MCI. Participants who had 24 to 27 points of Mini-Mental State Examination Japanese Version scores were diagnosed with amnestic MCI [31]. Participants were confirmed to have no arteriosclerotic dementia with magnetic resonance imaging or computed tomography scans performed within the previous 6 months. Each participant provided written informed consent after receiving a detailed explanation regarding the objectives and procedure of the study. This study consisted of a 12-week supplement ingestion period from August to December 2016. Participants with the following criteria were excluded from the study: 1) individuals who were taking medicine for cognitive impairment, 2) individuals who were taking supplements for improvement of cognitive function, 3) individuals who had been diagnosed with dementia according to the HDS-R, 4) individuals who had been diagnosed with depression according to the Japanese version of the Geriatric Depression Scale Short Form (GDS-S-J), 5) individuals who had impaired color vision, 6 ) individuals who had unequal function in the right and left hands due to injury or surgery, 7) individuals who were included or intended to participate in other trials which performed the oral administration of supplement or medicine, or percutaneous treatment with cosmetic or medicine, or who had participated in these kind of trials within one month, 8) individuals who had been classified as ineligible for this study by responsible doctor.

\section{Supplement formulation}

One AS capsule contained $3 \mathrm{mg}$ of astaxanthin, $5 \mathrm{mg}$ of sesamin, and other components including filling agents such as safflower oil, and dispersants. The placebo group received a placebo capsule that contained filling agents such as safflower oil, starch and water, dispersants, artificial colorants, but did not contain AS. Two capsules were administered every day for 12 weeks. AS capsules and placebo capsules were not distinguishable by their shape, taste, or color. The AS capsules contained natural astaxanthin derived from Haematococcus pluvialis (ASTOTS, FUJIFILM), which was processed using the dispersant technology that has been found to improve the absorbability of astaxanthin in humans [26]. We used sesame extract derived from Sesamum indicum. The dose of astaxanthin was determined by the previously performed study by other group, which evaluated the effect of $6 \mathrm{mg}$ or $12 \mathrm{mg}$ of astaxanthin on cognitive function in humans [17]. We decided the dose of sesamin by referring the previous study from another group, which evaluated the effect of $10 \mathrm{mg}$ of sesamin on fatigue in humans [32].

\section{Cognitive tests}

The CNSVS is a computerized test that evaluates multiple cognitive functions. CNSVS scores have been standardized according to the results from large populations of individuals aged 7-90 years [27]. The test can detect subtle cognitive changes that occur during aging or MCI [27, 33]. It evaluates 11 neuropsychological domains (composite memory, verbal memory, visual memory, processing speed, psychomotor speed, executive function, reaction time, complex attention, simple attention, cognitive flexibility, and motor speed) using 7 measures: verbal and visual memory tests, the finger tapping test, the symbol digit coding (SDC) test, the Stroop test, the shifting attention test, and the continuous performance test. In the finger tapping test, participants pressed the space key of a keyboard with their right or left index finger as many times as they could within 10 seconds. This test is known as a sensitive neuropsychological test that is used to identify brain impairments [27]. In the SDC test, the participants were given 120 seconds to type numbers that corresponded to a series of symbols shown on a screen. The participants first completed a training session in which they learned the link between the numbers and symbols by viewing a table showing the 
8 symbols above each of the 8 numbers. This table was in plain sight throughout the SDC test. In the trial, they typed numbers in empty boxes on the screen that corresponded to the symbols. This test is thought to be highly sensitive to cerebral dysfunction [27]. The details of the other tests are described in elsewhere [27]. The CNSVS test was performed at baseline and after 6 and 12 weeks of dietary supplementation.

The Japanese version of the ADAS-cog is one of the most popular tests of cognitive function [34]. The test is composed of 11 tasks that measure impairments in memory, language, praxis, attention, and other cognitive functions related to symptoms of $\mathrm{AD}$. The ADAS-cog test was performed at baseline and after 6 and 12 weeks of supplementation.

\section{Blood sampling and safety evaluation}

Serum and plasma were obtained from the participants at baseline, after 6 weeks, and after 12 weeks of dietary supplementation. MDA, ox-LDL, STAS, PON-1, and BDNF were analyzed by NIKKEN SEIL Co, Ltd for each time point. As a safety evaluation, we performed a general biochemical examination of blood and hematologic characteristics.

\section{Statistical analysis}

All results were presented as the mean \pm standard deviation (SD). The intra-group change from the baseline was evaluated using Dunnett's test. Differences between the placebo and AS groups were assessed by unpaired $t$-test. No additional analyses were performed. Probabilities less than $5 \%\left({ }^{*} p<0.05\right)$ were considered to be statistically significant. Statistical analyses were carried out using IBM SPSS statics (version 24).

\section{RESULTS}

\section{Participants}

Initially, we recruited 21 participants (aged 57-78, 14 males and 7 females) in the Tokyo metropoli$\tan$ area who had been diagnosed with MCI. The included participants were assigned to the AS group $(n=10)$ or placebo group $(n=11)$. One participant in the placebo group and two participants in the AS group discontinued the study due to withdrawal of consent for personal reasons. Three participants in the placebo group and one participant in the AS group were excluded from the analysis due to deviations from the protocol (Fig. 1). Finally, we analyzed data from 14 participants (aged 57-78, 9 males and 5 females). We performed per-protocol set analysis. There were no statistical differences between the initial and final groups of participants in terms of baseline data regarding age, BMI, GDSS-J, and HDS-R. The participants were recruited from August to September 2016. This study consisted of 12-week administration period from August to December 2016. The placebo group and AS group were matched according to age, gender, and BMI (Table 1). We observed no differences in baseline GDS-S-J and HDS-R scores between the placebo and AS groups. The average ingestion rates were not significantly different: $99.7 \pm 1.5 \%$ and $97.8 \pm 2.2 \%$ in the placebo and AS group, respectively. All subjects had an ingestion rate greater than $95 \%$.

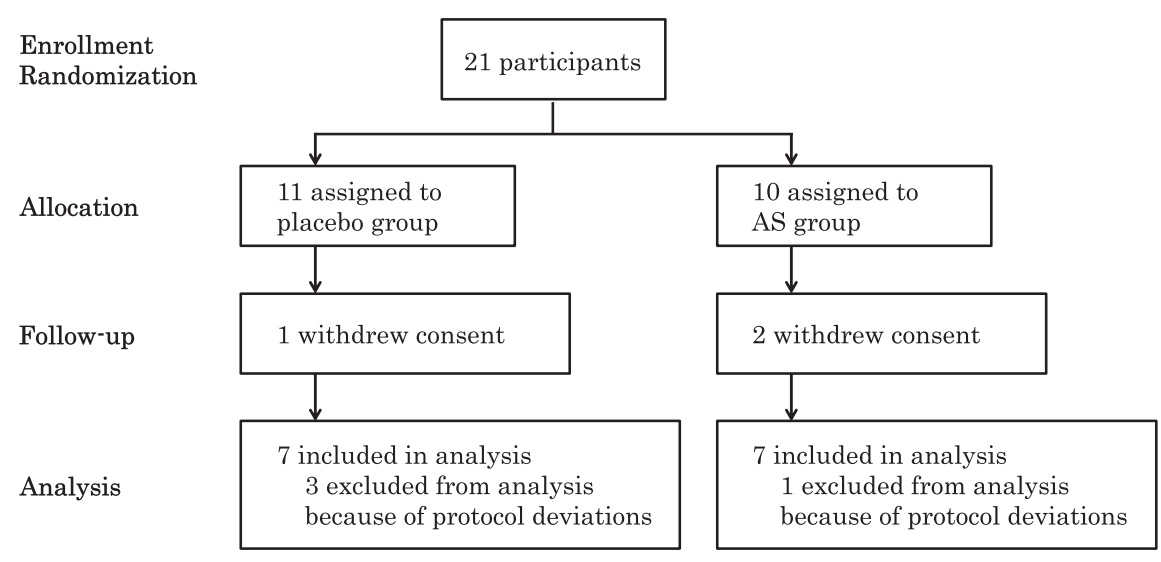

Fig. 1. Flow diagram of participants. AS, astaxanthin and sesamin. 


\section{Cognitive tests}

The aim of this study was to evaluate the effects of dietary supplementation with AS on cognitive functions. We performed the CNSVS and ADAS-cog tests at baseline, after 6 weeks, and after 12 weeks of supplementation. For the CNSVS test, we observed no statistically significant differences in the raw values between the AS and placebo groups (Table 2). However, the individuals in the AS group showed a significantly greater amount of change between the baseline and 12 weeks after supplementation in terms of psychomotor speed and processing speed, compared with individuals in the placebo group (Fig. 2). Furthermore, in the AS group, we observed a statistically significant increase in processing speed between the baseline and 12 weeks post-supplementation

Table 1

Baseline characteristics of participants who completed 12 weeks test

\begin{tabular}{lccc}
\hline & Placebo $(n=7)$ & AS $(n=7)$ & $p$ value \\
\hline Age $($ mean \pm SD) & $68.4 \pm 7.3$ & $70.4 \pm 7.0$ & 0.61 \\
Male, $n(\%)$ & $4(57.1)$ & $5(71.4)$ & \\
BMI (mean \pm SD) & $22.1 \pm 2.7$ & $21.6 \pm 3.4$ & 0.76 \\
GDS-S-J (mean \pm SD) & $5.43 \pm 3.7$ & $3.0 \pm 2.6$ & 0.18 \\
HDS-R (mean \pm SD) & $28.4 \pm 1.5$ & $29.3 \pm 1.1$ & 0.25 \\
\hline
\end{tabular}

$(p=0.018)$, although we observed no statistical difference in psychomotor speed $(p=0.133)$. For the ADAS-cog test, we observed no differences between the placebo and AS groups at any time points.

\section{Blood tests}

Extensive epidemiological research has demonstrated an association between the risk of cognitive decline and systemic oxidative stress [35]. To address this, we analyzed blood levels of MDA, ox-LDL, STAS, and PON-1 at baseline, after 6 weeks, and
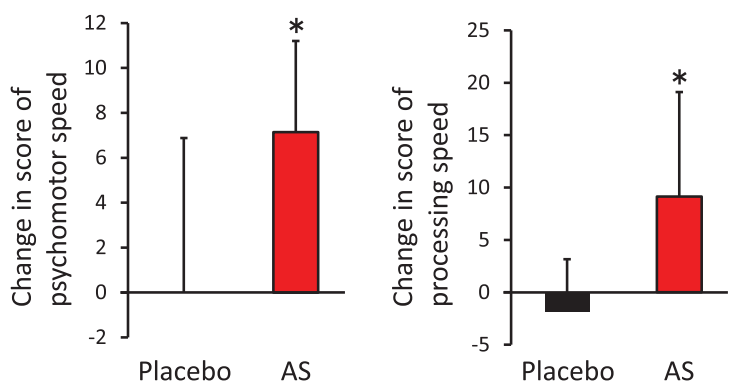

Fig. 2. Improvement of psychomotor speed and processing speed following dietary supplementation with astaxanthin and sesamin. Change in psychomotor speed (left) and processing speed (right) from baseline. ${ }^{*} p<0.05$ by unpaired $t$-test. Error bars indicate SD. AS, astaxanthin and sesamin.

Table 2

Score of cognitive tests

\begin{tabular}{lcccc}
\hline CNS Vital Signs domain & & & Week & \\
scores & & 0 & 6 & 12 \\
\hline \multirow{4}{*}{ Composite Memory } & Group & mean \pm SD & mean \pm SD & mean \pm SD \\
& Placebo & $94.0 \pm 16.7$ & $100.0 \pm 13.2$ & $93.7 \pm 16.6$ \\
Verbal Memory & AS & $98.1 \pm 17.9$ & $94.6 \pm 16.2$ & $89.0 \pm 12.9$ \\
& Placebo & $93.7 \pm 17.2$ & $98.0 \pm 17.7$ & $92.7 \pm 16.5$ \\
Visual Memory & AS & $92.3 \pm 14.1$ & $88.4 \pm 14.9$ & $90.0 \pm 17.0$ \\
& Placebo & $96.0 \pm 17.4$ & $103.1 \pm 9.0$ & $97.4 \pm 13.7$ \\
Psychomotor Speed & AS & $105.0 \pm 17.3$ & $103.4 \pm 15.2$ & $91.9 \pm 7.5$ \\
& Placebo & $104.0 \pm 14.1$ & $102.3 \pm 15.6$ & $104.0 \pm 9.3$ \\
Reaction Time & AS & $102.0 \pm 15.0$ & $102.7 \pm 17.4$ & $109.1 \pm 15.1$ \\
& Placebo & $87.0 \pm 17.8$ & $93.7 \pm 16.1$ & $91.0 \pm 11.1$ \\
Complex Attention & AS & $83.9 \pm 13.6$ & $83.6 \pm 15.2$ & $86.7 \pm 13.3$ \\
& Placebo & $99.3 \pm 10.8$ & $97.1 \pm 12.9$ & $109.1 \pm 11.5$ \\
Cognitive Flexibility & AS & $102.9 \pm 10.4$ & $104.0 \pm 6.6$ & $106.4 \pm 10.7$ \\
& Placebo & $91.7 \pm 12.4$ & $92.7 \pm 10.2$ & $102.7 \pm 11.9$ \\
Processing Speed & AS & $98.3 \pm 8.3$ & $98.0 \pm 7.4$ & $101.9 \pm 9.1$ \\
& Placebo & $11.9 \pm 10.3$ & $111.0 \pm 14.0$ & $110.0 \pm 8.4$ \\
Executive Function & AS & $105.7 \pm 14.6$ & $112.4 \pm 13.9$ & $114.9 \pm 16.1$ \\
& Placebo & $91.0 \pm 13.0$ & $94.6 \pm 11.7$ & $102.3 \pm 12.1$ \\
Simple Attention & AS & $98.4 \pm 7.9$ & $97.9 \pm 7.7$ & $102.6 \pm 8.7$ \\
& Placebo & $102.9 \pm 8.0$ & $96.9 \pm 18.3$ & $104.3 \pm 7.2$ \\
Motor Speed & AS & $101.7 \pm 14.7$ & $103.0 \pm 14.1$ & $98.9 \pm 15.6$ \\
& Placebo & $96.6 \pm 14.6$ & $95.1 \pm 13.4$ & $98.0 \pm 11.3$ \\
ADAS-cog & AS & $98.7 \pm 11.8$ & $95.9 \pm 15.0$ & $102.1 \pm 10.4$ \\
& Placebo & $3.00 \pm 1.75$ & $3.29 \pm 1.86$ & $2.27 \pm 1.22$ \\
& AS & $5.61 \pm 3.26$ & $4.27 \pm 2.76$ & $3.89 \pm 3.26$ \\
\hline
\end{tabular}


Table 3

List of adverse events

\begin{tabular}{lcc}
\hline & Placebo & AS \\
\hline Acute low back pain & 1 & 0 \\
Cold & 0 & 3 \\
Cystitis & 1 & 0 \\
Diarrhea & 0 & 1 \\
Dizziness & 0 & 1 \\
Feeling of smothering & 2 & 0 \\
Lassitude & 2 & 0 \\
Lip redness & 0 & 1 \\
Low back pain & 1 & 0 \\
Malaise & 1 & 0 \\
Occult blood in urine & 0 & 1 \\
Protein in urine & 0 & 1 \\
Slight cold & 0 & 1 \\
Sore throat & 0 & 1 \\
Stomatitis & 0 & 1 \\
Tonsillitis & 1 & 0 \\
\hline
\end{tabular}

after 12 weeks of supplementation. Higher serum BDNF levels were suggested to protect against future occurrence of dementia and $\mathrm{AD}$ [36]. Because administration of astaxanthin is known to alleviate oxidative stress-induced decreases in BDNF levels, we also analyzed blood levels of BDNF at baseline, after 6 weeks, and after 12 weeks of dietary supplementation [37]. We excluded the MDA results from the consideration because the statistical trend between the placebo and AS groups at baseline was observed $(0.87 \pm 0.29$ in placebo group versus $0.63 \pm 0.10$ in AS group, $p=0.054$ ). We did not observe statistically significant differences between the placebo and AS groups for ox-LDL, STAS, PON-1, or BDNF for any time points (data not shown).

\section{Clinical safety}

We observed several adverse events in both the placebo and AS groups (Table 3). There were no differences in the frequency of adverse events between the groups. We did not observe any adverse events related to the ingestion of AS. We found some statistically significant changes in the scores from the general biochemical blood examination and hematologic tests. However, all of these changes were slight and well within normal values. The responsible doctor confirmed that they did not represent a safety concern.

\section{DISCUSSION}

We found that dietary supplementation with AS for 12 weeks improved psychomotor speed and processing speed, as measured by the CNSVS test, which was related to the ability to comprehend, and perform complex tasks quickly and accurately $[38,39]$. Psychomotor speed is calculated as the sum of the finger tapping test and total correct responses in the SDC test. This reflects "How well a subject perceives, attends, responds to complex visual-perceptual information and performs simple fine motor coordination", and is related to "Ability preform simple motor skills and dexterity through cognitive functions, i.e., use of precision instruments or tools, performing mental and physical coordination, i.e., driving a car, playing a musical instrument" [27, 38]. Processing speed is calculated by subtracting the number of errors from the correct responses in the SDC test. Processing speed reflects "How well a subject recognizes and processes information i.e., perceiving, attending/responding to incoming information, motor speed, fine motor coordination, and visual-perceptual ability", and is related to "Ability to recognize and respond/react, i.e., fitness-to-drive, occupation issues, possible danger/risk signs or issues with accuracy and detail" [27, 38]. The majority of clinical trials evaluating the effects of supplements on cognitive function have focused on memory improvement. Thus, the lack of improved verbal memory or visual memory observed in the AS group may indicate a unique effect of AS dietary supplementation on cognitive function, distinguishing it from other supplements such as Ginkgo Biloba [40]. Although astaxanthin can cross the blood-brain barrier [11], its detailed distribution in the brain is unknown. Because carotenoids show inclined distribution in some tissue [41], analysis of the detailed distribution of astaxanthin and its bioactivity in the brain merits future investigation to explain the unique role of AS on cognitive function, because both psychomotor speed and processing speed are related to frontal lobe function. Recently, the presence of brain amyloids in earlier stages of MCI was associated with increased safety risks of driving [42]. Because psychomotor speed is related to mental and physical coordination, which are required for using some instrument or driving a car [39, 43], AS dietary supplementation might be useful for reducing the risk of car accidents in the aging Japanese population. In addition, AS supplementation will improve the ability of elderly people to quickly and accurately judge complex information, which is related to processing speed.

To our knowledge, this study is the first to examine the effects of combined supplementation of AS 
on cognitive functions. The effects of dietary supplementation with astaxanthin only on the improvement of cognitive functions has been previously performed using Coghealth, although statistically significant differences between the placebo and astaxanthin groups were not observed [17]. As described in the Materials and Methods section, we used the dispersant technology to enhance the absorbability of astaxanthin in humans [26]. This technology appears to have reinforced the effects of astaxanthin on cognitive functions. Indeed, astaxanthin has a neuroprotective effects in that it reduces oxidative stress, and has been found to alleviate oxidative stress-related brain dysfunctions, improve learning or memory, prevent neurodegeneration, cellular toxicity, or apoptosis of neurons, and promote cellular survival [17-19, 44-46]. In addition to neuroprotective effects, administration of astaxanthin enhanced adult hippocampal neurogenesis and spatial memory in mice [47]. Administration of astaxanthin also prevented depression by inhibiting hippocampal inflammation in diabetic mice [48]. Furthermore, the activities of endogenous anti-oxidative enzymes were upregulated by treatment with astaxanthin, suggesting that astaxanthin acted as antioxidant both by anti-oxidative capacity itself and by activating endogenous anti-oxidative capacity [49]. In addition to astaxanthin, administration of sesamin has been found to have the neuroprotective effects in cerebral ischemia or traumatic brain injury [21, 22, 50]. Sesamin also prevented the inflammation in co-culture of microglia and neuron [51], or protect PC12 cells from high glucose-induced oxidation and apoptosis [52]. Sesamin and its metabolites are also known to promote neuronal differentiation [53]. These pleiotropic effects of AS might be involved in the improvement of cognitive function. In addition to the independent effects of astaxanthin or sesamin, we hypothesized that the cognitive functions were improved by the synergistic effects of AS, as described in Introduction section. The between-group differences observed in this study reinforced our hypothesis. However, the effects of sesamin on cognitive function have not been investigated in humans. Thus, we could not conclude that the improvement of cognitive functions observed in this study was derived from astaxanthin with enhanced absorbability, sesamin, or synergistic effects of AS.

A previous extensive epidemiological study showed that high levels of thiobarbituric acid-reactive substances that reflected the level of lipid peroxidation were correlated with the risk of cognitive decline [35]. The level of lipid peroxidation in human erythrocytes has been found to be reduced by administration of astaxanthin [9]. Furthermore, astaxanthin is known to cross the blood-brain barrier, and exert a neuroprotective effect by reducing oxidative stress or increasing the BDNF level [11, 37, 54]. We therefore expected administration of AS to decrease systemic levels of oxidative stress, and to increase levels of BDNF. Although we did not observe systemic changes in STAS, PON-1, or BDNF levels, reduced oxidative stress or increased levels of BDNF in microenvironments in the brain might have been involved in the observed improvement of cognitive functions.

Our study has several limitations. Even though our sample size was limited and we performed perprotocol set analysis which rated this trial as pilot study, we observed AS-induced improvements in psychomotor speed and processing speed, suggesting that AS have strong effects on cognitive functions. Astaxanthin is found in foods such as shrimp, salmon, and crab. These types of seafood, along with sesame, are important elements of Japanese cuisine. In this study, we did not estimate participant dietary intake of astaxanthin and sesame, leading to a possible over- or under-estimation of the effects of AS. We hypothesized that combined supplementation of AS exerted much stronger anti-oxidative activity than single supplementation. In fact, we observed the improvement of cognitive functions. However, administration of AS led to no observable changes in systemic levels of oxidative stress or BDNF. Thus, precise mechanisms how AS improved cognitive functions were not elucidated. Further analysis is required to examine how AS influence cognitive functions.

\section{ACKNOWLEDGMENTS}

We thank Mr. Mutsuki Kikawada (Memory Clinic Ochanomizu) for valuable help with study execution.

Authors' disclosures available online (https:// www.j-alz.com/manuscript-disclosures/17-0969r1).

\section{REFERENCES}

[1] World Health Organization, Medical centre, Fact sheets, Dementia, http://www.who.int/mediacentre/factsheets/fs 362/en, Last updated 2017, Accessed on 2017.

[2] Petersen RC (2000) Mild cognitive impairment: Transition between aging and Alzheimer's disease. Neurologia 15, 93-101. 
[3] Swomley AM, Butterfield DA (2015) Oxidative stress in Alzheimer disease and mild cognitive impairment: Evidence from human data provided by redox proteomics. Arch Toxicol 89, 1669-1680.

[4] Maurya PK, Kumar P, Nagotu S, Chand S, Chandra P (2016) Multi-target detection of oxidative stress biomarkers in quercetin and myricetin treated human red blood cells. RSC Adv 6, 53195-53202.

[5] Cao L, Tan L, Wang H-F, Jiang T, Zhu X-C, Lu H, Tan MS, Yu J-T (2016) Dietary patterns and risk of dementia: A Systematic review and meta-analysis of cohort studies. $\mathrm{Mol}$ Neurobiol 53, 6144-6154.

[6] Hussein G, Sankawa U, Goto H, Matsumoto K, Watanabe $\mathrm{H}$ (2006) Astaxanthin, a carotenoid with potential in human health and nutrition. J Nat Prod 69, 443-449.

[7] Ambati RR, Moi PS, Ravi S, Aswathanarayana RG (2014) Astaxanthin: Sources, extraction, stability, biological activities and its commercial applications - A review. Mar Drugs 12, 128-152.

[8] Mori J, Yokoyama H, Sawada T, Miyashita Y, Nagata K (2013) Anti-oxidative properties of astaxanthin and related compounds. Mol Cryst Liq Cryst 580, 52-57.

[9] Nakagawa K, Kiko T, Miyazawa T, Carpentero Burdeos G, Kimura F, Satoh A, Miyazawa T (2011) Antioxidant effect of astaxanthin on phospholipid peroxidation in human erythrocytes. Br J Nutr 105, 1563-1571.

[10] Komatsu T, Sasaki S, Manabe Y, Hirata T, Sugawara T (2017) Preventive effect of dietary astaxanthin on UVAinduced skin photoaging in hairless mice. PLoS One 12, 1-16.

[11] Tso M, Lam T (1996) Method of Retarding and Ameliorating Central Nervous System and Eye Damage. US Patent. 5527533.

[12] Tominaga K, Hongo N, Karato M, Yamashita E (2012) Cosmetic benefits of astaxanthin on humans subjects. Acta Biochim Pol 59, 43-47.

[13] Yoshida H, Yanai H, Ito K, Tomono Y, Koikeda T, Tsukahara H, Tada N (2010) Administration of natural astaxanthin increases serum HDL-cholesterol and adiponectin in subjects with mild hyperlipidemia. Atherosclerosis 209, 520-523.

[14] Ni Y, Nagashimada M, Zhuge F, Zhan L, Nagata N, Tsutsui A, Nakanuma Y, Kaneko S, Ota T (2015) Astaxanthin prevents and reverses diet-induced insulin resistance and steatohepatitis in mice: A comparison with vitamin E. Sci $\operatorname{Rep} \mathbf{5}, 17192$.

[15] Earnest CP, Lupo M, White KM, Church TS (2011) Effect of astaxanthin on cycling time trial performance. Int J Sports Med 32, 882-888.

[16] Saito H, Cherasse Y, Suzuki R, Mitarai M, Ueda F, Urade Y (2017) Zinc-rich oysters as well as zinc-yeast- and astaxanthin-enriched food improved sleep efficiency and sleep onset in a randomized controlled trial of healthy individuals. Mol Nutr Food Res 61, 1600882.

[17] Katagiri M, Satoh A, Tsuji S, Shirasawa T (2012) Effects of astaxanthin-rich Haematococcus pluvialis extract on cognitive function: A randomised, double-blind, placebocontrolled study. J Clin Biochem Nutr 51, 102-107.

[18] Wu H, Niu H, Shao A, Wu C, Dixon BJ, Zhang J, Yang S, Wang Y (2015) Astaxanthin as a potential neuroprotective agent for neurological diseases. Mar Drugs 13, 5750-5766.

[19] Grimmig B, Kim SH, Nash K, Bickford PC, Douglas Shytle R (2017) Neuroprotective mechanisms of astaxanthin: A potential therapeutic role in preserving cognitive function in age and neurodegeneration. GeroScience 39, 19-32.
[20] Asghar A, Majeed MN, Akhtar MN (2014) A review on the utilization of sesame as functional food. Am J Food Nutr 4, 21-34

[21] Cheng FC, Jinn TR, Hou RC, Tzen JT (2006) Neuroprotective effects of sesamin and sesamolin on gerbil brain in cerebral ischemia. Int J Biomed Sci 2, 284-288.

[22] Guo H, Tian J, Wang X, Tian Z, Li X, Yang L, Zhao M, Liu S (2015) Neuroprotection of sesamin against cerebral ischemia in-vivo and N-Methyl-D-Aspartate-induced apoptosis in-vitro. Biochem Pharmacol (Los Angel) 4, 185.

[23] Petri D, Lundebye A-K (2007) Tissue distribution of astaxanthin in rats following exposure to graded levels in the feed. Comp Biochem Physiol C Toxicol Pharmacol 145, 202-209.

[24] Tomimori N, Rogi T, Shibata H (2017) Absorption, distribution, metabolism, and excretion of [(14) C]sesamin in rats. Mol Nutr Food Res 61, doi: 10.1002/mnfr.201600844

[25] Umeda-Sawada R, Ogawa M, Igarashi O (1999) The metabolism and distribution of sesame lignans (sesamin and episesamin) in rats. Lipids 34, 633-637.

[26] Sato M (2017) Japan patent. P2017-012059A.

[27] Gualtieri CT, Johnson LG (2006) Reliability and validity of a computerized neurocognitive test battery, CNS Vital Signs. Arch Clin Neuropsychol 21, 623-643.

[28] Mohs RC, Rosen WG, Davis KL (1983) The Alzheimer's disease assessment scale: An instrument for assessing treatment efficacy. Psychopharmacol Bull 19, 448-450.

[29] Udani JK (2013) Effects of superulam on supporting concentration and mood: A randomized, double-blind, placebo-controlled crossover study. Evid Based Complement Alternat Med 2013, 238454.

[30] Ayano I, Yuriko O, Shinobu S, Hiroyuki S, Takashi K, Koh M, Masami S (2016) Effects of capsule containing astaxanthin on skin condition in healthy subjects -a randomized, double-blind, parallel-group, placebo-controlled study. Jpn Pharmacol Ther 44, 1209-1216.

[31] Petersen RC (2004) Mild cognitive impairment as a clinical entity and treatment target. Arch Neurol 62, 1160-1163; discussion 1167.

[32] Takemoto D, Yasutake Y, Tomimori N, Ono Y, Shibata H, Hayashi J (2015) Sesame lignans and vitamin E supplementation improve subjective statuses and anti-oxidative capacity in healthy humans with feelings of daily fatigue. Glob J Health Sci 7, 1-10.

[33] Gualtieri CT (2005) Neurocognitive testing supports a broader concept of mild cognitive impairment. $A m J$ Alzheimers Dis Other Demen 20, 359-366.

[34] Kodama C, Noguchi H, Kinoshita T, Hidaka S, Sasaki M, Yamashita F, Asada T (2005) The Alzheimer's disease Assessment Scale - Cognitive Subscale (Japanese version): The performance in community-dwelling people with mild cognitive impairment. Alzheimers Dement 1, S25-S26.

[35] Berr C, Balansard B, Arnaud J, Roussel AM, Alpérovitch A (2000) Cognitive decline is associated with systemic oxidative stress: The EVA study. Etude du Vieillissement Artériel. J Am Geriatr Soc 48, 1285-1291.

[36] Weinstein G, Beiser AS, Choi SH, Preis SR, Chen TC, Vorgas D, Au R, Pikula A, Wolf PA, DeStefano AL, Vasan RS, Seshadri S (2014) Serum brain-derived neurotrophic factor and the risk for dementia: The Framingham Heart Study. JAMA Neurol 71, 55-61.

[37] Wu W, Wang X, Xiang Q, Meng X, Peng Y, Du N, Liu Z, Sun Q, Wang C, Liu X (2014) Astaxanthin alleviates brain aging in rats by attenuating oxidative stress and increasing BDNF levels. Food Funct 5, 158-166. 
[38] CNS Vital Signs. Brief Interpretation Guide. http://www. cnsvs.com/WhitePapers/CNSVS-BriefInterpretation Guide.pdf

[39] Cognitrax. Brief Interpretation Guide. http://www.cogni trax.com/Manuals/CognitraxBriefInterpretationGuide.pdf

[40] Santos RF, Galduróz JCF, Barbieri A, Castiglioni ML V, Ytaya LY, Bueno OFA (2003) Cognitive performance, SPECT, and blood viscosity in elderly non-demented people using Ginkgo biloba. Pharmacopsychiatry 36, 127-133.

[41] Rao AV, Rao LG (2007) Carotenoids and human health. Pharmacol Res 55, 207-216.

[42] Ott BR, Jones RN, Noto RB, Yoo DC, Snyder PJ, Bernier JN, Carr DB, Roe CM (2017) Brain amyloid in preclinical Alzheimer's disease is associated with increased driving risk. Alzheimers Dement (Amst) 6, 136-142.

[43] Davis RL, Ohman JM (2017) Driving in early-stage Alzheimer's disease: An integrative review of the literature. Res Gerontol Nurs 10, 86-100.

[44] Liu X, Shibata T, Hisaka S, Osawa T (2009) Astaxanthin inhibits reactive oxygen species-mediated cellular toxicity in dopaminergic SH-SY5Y cells via mitochondria-targeted protective mechanism. Brain Res 1254, 18-27.

[45] Chang C-H, Chen C-Y, Chiou J-Y, Peng RY, Peng C-H (2010) Astaxanthine secured apoptotic death of PC12 cells induced by beta-amyloid peptide 25-35: Its molecular action targets. J Med Food 13, 548-556.

[46] Xue Y, Qu Z, Fu J, Zhen J, Wang W, Cai Y, Wang W (2017) The protective effect of astaxanthin on learning and memory deficits and oxidative stress in a mouse model of repeated cerebral ischemia/reperfusion. Brain Res Bull 131, 221-228.

[47] Yook JS, Okamoto M, Rakwal R, Shibato J, Lee MC, Matsui T, Chang HK, Cho JY, Soya H (2016) Astaxanthin supplementation enhances adult hippocampal neurogenesis and spatial memory in mice. Mol Nutr Food Res 60, 589-599.

[48] Zhou XY, Zhang F, Hu XT, Chen J, Tang RX, Zheng KY, Song YJ (2017) Depression can be prevented by astaxanthin through inhibition of hippocampal inflammation in diabetic mice. Brain Res 1657, 262-268.

[49] Lee DH, Kim CS, Lee YJ (2011) Astaxanthin protects against MPTP/MPP+-induced mitochondrial dysfunction and ROS production in vivo and in vitro. Food Chem Toxicol 49, 271-280.

[50] Liu Y, Xu Z, Yang G, Yang D, Ding J, Chen H, Yuan F, Tian H (2017) Sesamin alleviates blood-brain barrier disruption in mice with experimental traumatic brain injury. Acta Pharmacol Sin 38, 1445-1455.

[51] Bournival J, Plouffe M, Renaud J, Provencher C, Martinoli MG (2012) Quercetin and sesamin protect dopaminergic cells from MPP+-induced neuroinflammation in a microglial (N9)-neuronal (PC12) coculture system. Oxid Med Cell Longev 2012, 921941.

[52] Bournival J, Francoeur M-A, Renaud J, Martinoli M-G (2012) Quercetin and sesamin protect neuronal PC12 cells from high-glucose-induced oxidation, nitrosative stress, and apoptosis. Rejuvenation Res 15, 322-333.

[53] Hamada N, Fujita Y, Tanaka A, Naoi M, Nozawa Y, Ono Y, Kitagawa Y, Tomimori N, Kiso Y, Ito M (2009) Metabolites of sesamin, a major lignan in sesame seeds, induce neuronal differentiation in PC12 cells through activation of ERK1/2 signaling pathway. J Neural Transm 116, 841-852.

[54] Lee DH, Lee YJ, Kwon KH (2010) Neuroprotective effects of astaxanthin in oxygen-glucose deprivation in SH-SY5Y cells and global cerebral ischemia in rat. J Clin Biochem Nutr 47, 121-129. 